for the isotopic abundance of samarium-146 can be calculated from the results obtained here. An upper limit of $2 \times 10^{-7}$ per cent for the isotopic abundance of samarium-146 in natural samarium was calculated. The best mass spectrometric limit, obtained by Collins, Rourke and White, is $8 \times 10^{-5}$ per cent (ref. 5).

Recently, the $\alpha$-particle energy of samarium-146 (produced by bombardment of neodymium with $\alpha$-particles) was rechecked and found to agree exactly with the value Seaborg and Dunlavey originally reported $(2.55 \mathrm{MeV}$.) (M. C. Michel and R. D. Macfarlane, Lawrence Radiation Laboratory, unpublished results). From theoretical considerations based on this energy, an $\alpha$-half-life in the neighbourhood of $6 \times 10^{7}$ years is favoured. If this is the correct halflife, all primordial samarium-146 should now be neodymium-142.

The co-operation of the Oak Ridge National Laboratory in specially preparing the samarium sample is gratefully acknowledged. This research was supported by the U.S. Atomic Energy Commission.

Ronatd D. Macfartane

Lawrence Radiation Laboratory,

University of California,

Berkeley, California.

${ }^{1}$ Dunlavey, D. C., and Seaborg, G. T., Phys. Rev., 92, 206 (1953).

"Macfarlane, R. D., "Natural Alpha Radioactivity in Medium-Heavy Elements" (thesis), Department of Chemistry, Carnegie Institut of Technology, $N Y O-7687$ (May 1959).

"Macfarlane, R. D., and Kohman, T. P., "Natural Alpha Radioactivity in Medium-Heavy Elements" (in the press).

+ Vorob'ev, A. A., Komar, A. P., Korolev, V. A., and Slogakin, G. E. J. Exp. Theor. Phys. (U.S.S.R.), 37, 546 (1959); Soviet PhysicsJETP, 37, $386(1960)$.

"Collins, T. L., Rourkr, F, M., and White, F. A., Phys. Rev., 105, 196 (1957).

\section{Radiation-induced Defects in Lead Silicate Glass}

IN attempts to explain the process of coloration of glass by $\gamma$-radiation, it was suggested in a previous communication ${ }^{1}$ that electrons are trapped in defects to form colour centres. Not only do these defects already exist in glass but they can also be created by exposure to radiation. If defects could not be so created, it would be expected that the concentration of colour centres, as measured by the optical density, would increase exponentially to a saturation value at a certain dose. In practice, it is found that the optical density increases exponentially with dose up to about $10^{5.5}$ rads, but thereafter the increase is linear. This effect is shown in Fig. I, which is divided into two sections having different scales for dose, the first section illustrating the exponential portion and the second the linear portion of the curve.

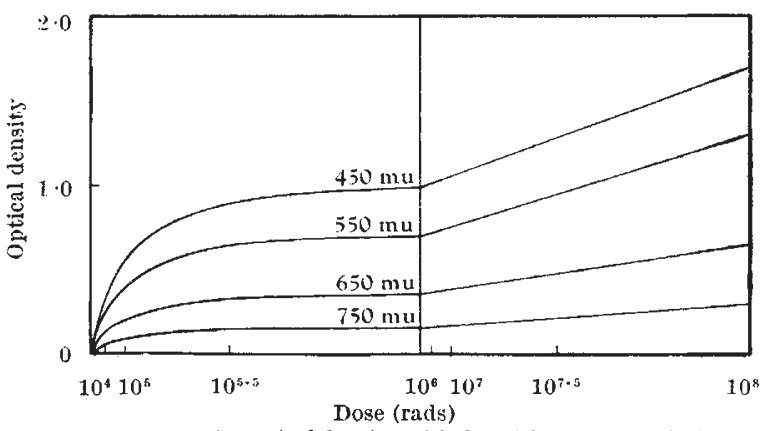

Fig. 1. Variation in ontical density with dose (tine after radiation, 1,000 min.)

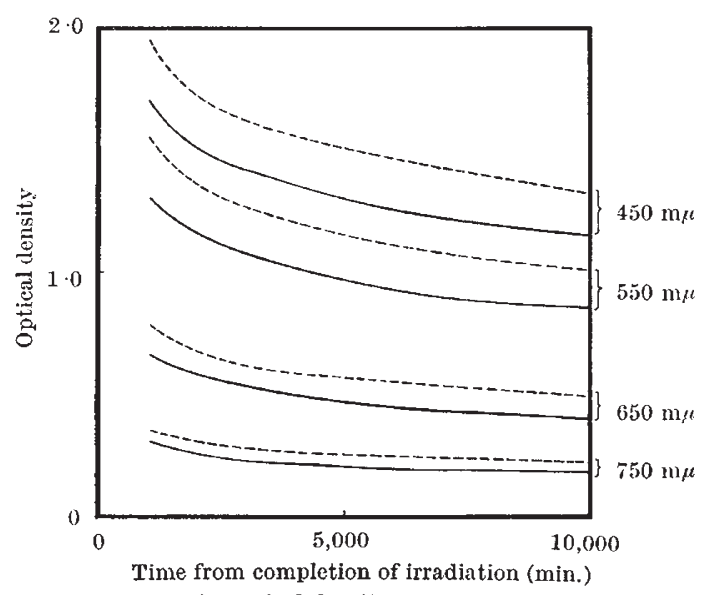

Fig. 2. Increase in optical density on second irradiation. -
first irradiation; - - , second irradiation after bleaching

The linear portion is attributed to the creation of defects by the radiation. Thus the extrapolation of the straight line to zero dose gives an indication of the defects already present in the glass. The differ. ence between the extrapolated value at zero dose and the measured optical density is proportional to the concentration of defects produced by the radiation.

Exposure of irradiated glass to light at room temperature produces bleaching of the colour centres by excitation of the trapped electrons to such energy. levels as to enable them to escape from the defects and recombine with the positive holes. It might be expected that such treatment should release the trapped electrons but have little effect on the total number of defects. Hence such a bleached sample of glass should contain a greater concentration of defects or trapping centres than an unirradiated sample and, on further irradiation, should contain more colour centres than an originally unirradiated sample which is subjected to a first irradiation.

To test this theory a sample of the lead silicate glass used in the experiments described previously was given a dose of $10^{8}$ rads of $\gamma$-radiation and its optical density was measured over the wave-length range $360-1,000 \mathrm{~m} \mu$ at intervals of time during the weck following the irradiation. The induced colora. tion was then bleached by exposure to light from a mercury lamp until the optical density of the glass was not significantly different from that measured before the irradiation. The sample was then given a further dose of $10^{8}$ rads, and the optical absorption was again measured.

Fig. 2 shows the variation in optical density with time for four wave-lengths after the first and second irradiations. It can be seen that the optical density of the sample after bleaching and re-irradiation is at all times greater than that obtained after the first irradiation. This result is in accord with the proposed theory.
R. S. BARKER
D. A. RICHARDSON
E. A. G. MCConkey
R. E. YEADON

Research Laboratories,

Pilkington Brothers, Ltd., St. Helens,

Lancashire.

${ }^{1}$ Barker, R. S., Richardson, D. A., McConkey, E. A. G., and Rimmer R., Nature, 187, 135 (1960). 